\title{
Patmos, entre la révélation du sacré et l'opacité du quotidien : Hölderlin, Papaditsas, Lorand Gaspar
}

Lito loakimidou

\section{OpenEdition}

\section{Journals}

Édition électronique

URL : http://journals.openedition.org/transtexts/219

DOI : 10.4000/transtexts. 219

ISSN : 2105-2549

Éditeur

Gregory B. Lee

Édition imprimée

Date de publication : 1 janvier 2008

Pagination : 61-80

ISSN : 1771-2084

\section{Référence électronique}

Lito loakimidou, «Patmos, entre la révélation du sacré et l'opacité du quotidien : Hölderlin, Papaditsas, Lorand Gaspar », Transtext(e)s Transcultures 跨文本跨文化 [En ligne], Hors série | 2008, mis en ligne le 14 septembre 2009, consulté le 10 décembre 2020. URL : http://journals.openedition.org/transtexts/ 219 ; DOI : https://doi.org/10.4000/transtexts.219

() Tous droits réservés 


\title{
Patmos, entre la révélation du sacré et l'opacité du quotidien : Hölderlin, Papaditsas, Lorand Gaspar.
}

\author{
LITO IOAKIMIDOU \\ Aristotle University - Thessaloniki
}

\begin{abstract}
This article evaluates through a comparative process the mythologizing of Patmos, island of the Apocalypse, by Friedrich Hölderlin, D. P. Papaditsas and Lorand Gaspar. This "recently created" myth is first examined through the scraps of narrative remaining in the different representations describing the initiation/visit of this space, a visit that assumes the characteristics of a fantasized journey inside mankind's religious history, or of a deathly peripeteia of the mind, or again of a frugal and elliptical deepening of the innermost self/ space / universe. The antonymic features of this experiment and of its verbal expression are subsequently studied, leading to emergence of yet another founding myth to be added to the long list of myths about humankind's writing, a superior stage of this artistic practice through the elaboration of the condensed, syncretic, fragile and sporadic message of the insular space.
\end{abstract}

Le mythe littéraire, dans son acception polymorphe à travers les âges et les différentes conceptions du phénomène littéraire, pose le problème des limites de son étendue, des circonstances de son émergence et surtout des conditions dans lesquelles il sera conçu et reçu comme tel par le lecteur. Sanctifier, sublimer le vécu est peut-être le propre de la poésie: le résultat n'en est pas pour autant le surgissement d'un mythe littéraire, notion qui jouit d'un substrat culturel trop large 
pour être compris comme synonyme de ce qui serait plutôt un mythe personnel, un élément signifiant pour le trajet intellectuel du créateur, avec ou sans accompagnement d'interprétation psychanalytique. Par contre, le mythe littéraire, outre la liaison privilégiée qu'il entretient avec la notion de récit, même décomposée jusqu'au désordre chaotique, se fonde sur un nombre suffisant de réitérations esthétiques, tel qu'il puisse établir un lien incontestable entre le noyau sémantique du mythe, sa présence axiomatique, pourrait-on dire, et la résurgence d'un ou de plusieurs de ses mythèmes, suivant la logique de la partie qui, même sous un travestissement idéologique, formel, générique, renvoie au centre d'un tout irréductible. L'acceptation de ce cadre théorique fait plonger dans l'indécision la réception du lecteur qui aborde la prose de Papaditsas consacrée à l'autocommentaire de son recueil A Patmos et qui, écrite en 1964, l'année même de la publication du recueil, élève $l^{\prime}$ " expérience de Patmos», du nom que le poète donne à sa rencontre avec l'île de l'Apocalypse, au niveau d'un nouveau mythe, un mythe " récemment forgé », selon les paroles de Papaditsas. ${ }^{1}$ La présente étude, qui veut évaluer le bien-fondé d'une telle équation, une association mentale du mythe à l'action de voir une île, de la contempler, de la conceptualiser par l'intellect et d'en parler par la voie poétique, inclut donc l'examen de trois récurrences de Patmos dans la littérature, dont chacune jouit d'un substrat culturel et d'une expression esthétique complètement différents. L'Allemand Friedrich Hölderlin, quelques années avant de sombrer définitivement dans l'aliénation mentale, compose, dans le début des années 1800, ses derniers grands hymnes, parmi lesquels l'hymne géométrique (quinze strophes de quinze vers) Patmos. Consacré à un espace que le poète n'a jamais visité, même s'il a déjà eu recours à la riche littérature de voyage pour composer, quelques années avant, le paysage grec de son roman Hypérion, Patmos devient le cadre de la réapparition, intermittente et mystique, du divin, à travers Jésus-Christ, selon toutefois la conception syncrétique et polythéiste du sacré que prône Hölderlin. ${ }^{2}$ En 1964, le poète grec Dimitris Papaditsas fait de Patmos, selon ses propres termes, «un topos, un trope et une rupture dans [sa] poésie ». Ce recueil, composé de onze parties suivies d'une conclusion poétique, en vers libres, retrace «un topos au-dessous duquel, sous une voûte de pierre, le temps et les sensations se renversent et, comme ionisés par le rêve, se transforment

\footnotetext{
${ }^{1}$ D. P. Papaditsas, A Patmos, 1964, dans Poésie 2, Athènes, éditions Gnossi, 1981 (en grec). C'est d'après cette édition que nous traduisons les extraits utilisés dans cet article. Le recueil est précédé d'une courte introduction « explicative », qui synthétise l'expérience du poète. Nous nous servons aussi des textes en prose de Papaditsas,

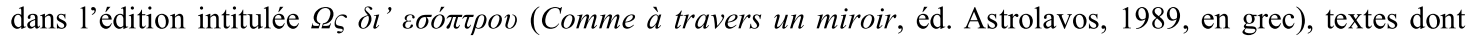
l'aspect de commentaire est toujours sublimé en inspiration vitale.

${ }^{2}$ Friedrich Hölderlin, Euvre poétique complète, texte établi par Michel Knaupp, traduit de l'allemand par François Garrigue, Paris, éditions de la Différence, 2005. Dans les années 1803-1806, Patmos a été remanié à plusieurs reprises. Nous étudions le texte d'après la version de 1803, dédiée au Landgrave de Hombourg, p. $824-835$ de l'édition citée.
} 
en présent dilaté et en révélations $»{ }^{3}$ Deux ans après, par le recueil Deux interprétations, il ajoute à ce torrent d'images surréalistes exprimées dans une langue abrupte et condensée, une expression plus ample, où l'action poétique de voir et d'interpréter est prise aux filets d'une tonalité apocalyptique. ${ }^{4}$ Enfin, Lorand Gaspar, dans le recueil paru en 1989, cristallise sa propre « expérience de Patmos », que le lecteur peut décoder à l'aide de certaines pages des Feuilles d'observation et des Carnets consacrés à cette île. ${ }^{5}$ Quant au recueil lui-même, composé de dix-huit pièces relativement ou très brèves (de trois à vingt vers), il simplifie l'expérience de l'île en sensations qui, correspondant aux éléments de base du paysage, se fondent l'une dans l'autre et s'enchevêtrent dans une métaphore au sens propre du terme, puisque le lecteur se voit transporté de la vue au toucher, de l'impression tactile à l'audible et ainsi de suite, le poète palpant sans cesse les éléments constitutifs de l'univers.

Ainsi, dans les trois expressions poétiques de l'île de l'Apocalypse, le sacré, ici immanent, là transcendantal, est incontestablement présent. Encore faut-il voir l'aventure de ses multiples apparitions, une aventure qui a ses épisodes, ses mythèmes, serait-on tenté de dire, privilégiés, une aventure qui métamorphose l'endroit géographique donné en réalité progressivement sublimée et l'espace fermé, isolé, délinéé d'une île en "théâtre", en "scène" où se joue une pièce contradictoire, celle de la présence et de la disparition, circulaires, répétitives, d'un sens à cerner et à proférer.

Comme on peut s'y attendre, dans le cas d'un univers clos et compact, dense de sens, qu'est celui d'une île, Patmos est le bout d'un voyage qui, même dans sa reformulation la plus spirituelle, conserve un minimum d'étapes progressives; ces bribes d'une narrativité mythologique de plus en plus estompée dans la poésie servent logiquement à garantir la réception du passage générique du mythe, dont l'unité est à recomposer par le lecteur. En étudiant les rapports entre la symbolique insulaire et l'espace poétique, Z. I. Siaflékis applique le terme d'identité narrative, proposé par Paul Ricoeur, à l'espace méditerranéen, elliptiquement décrit par le verbe poétique ; cette narrativité problématique apporte une polyvalence à explorer tant par le producteur que par le récepteur du message, et cela d'autant plus impérativement que « la représentation poétique d'un élément géographique est un

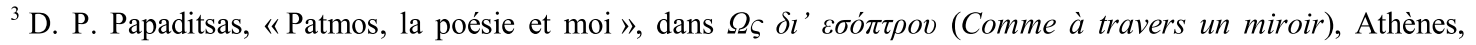
éditions Astrolavos, 1989 (en grec, texte de 1964).

${ }^{4}$ Le recueil A Patmos est réédité en 1966, suivi de Deux interprétations. Dans l'édition de Gnossi (1981), les deux ensembles poétiques, séparés, sont toutefois contigus.

${ }^{5}$ Lorand Gaspar, Patmos (1989), repris dans Patmos et autres poèmes, NRF Poésie/Gallimard, 2004. Les Feuilles d'observation (NRF Gallimard, 1986) contiennent les Carnets de Patmos (p. 71 sqq), à part quelques pages incluses dans Egée Judée, NRF Poésie/Gallimard, 1993, sous le titre de Journal de Patmos.
} 
processus qui inclut plusieurs conditions et fonctions : représentabilité, travail de la mémoire, reconstitution ésotérique $d u$ lieu, autoréférentialité. Les îles méditerranéennes, et notamment les grecques, soumettent leur pouvoir de représentabilité, mais aussi de mémorisation à leurs récepteurs, à cause précisément de la forte relation mythe-nature qui marque leur existence $» .{ }^{6}$ Or la mythification du paysage insulaire de Patmos ne correspond pas à un schéma mythique préexistant (à moins que nous ne l'identifiions à l'événement de l'Apocalypse ; cependant nous allons voir que le «mythe de Patmos » dépasse le cadre religieux de cette clôture biblique). Nous avons donc affaire à un mythe de et à travers la poésie, un schéma mythique qui se forge, se tord, s'estompe et réapparaît au fur et à mesure que le poète accomplit sa visite de l'île tout en donnant un cours créatif à sa réaction. En tout cas, cette action minimale insinue parfois même le danger mortel de toute initiation : surtout si, à l'image des « fils des Alpes » qui passent sans peur " par-dessus l'abîme / sur ponts de structure légère », le poète allemand doit vivre de fond en comble l'expérience de dépaysement, dédoublée par l'élévation immatérielle dans l'éther, qui donne accès à une vue vertigineuse, suivant la métamorphose de l'espace. En effet, des trois versions poétiques, celle de Hölderlin accorde une place remarquable au voyage d'approche de Patmos, sans doute parce que le poète est préoccupé, dans la dernière étape de sa production, par la manière de joindre l'espace conceptualisé de sa patrie symbolique, la Grèce, et la Germanie, patrie réelle.7 Le premier tiers des quinze strophes est donc consacré à ce trajet, qui, dans un premier temps, semble schématiser l'opposition Europe - Asie, l'espace germanique avec les " cimes du temps », " les monts écartelés », le " clair - obscur [d'où sortent] pâles sur mon passage, / La forêt ombreuse / Et les ruisseaux nostalgiques / Du pays »d'une part et, d'autre part, la luminosité aveuglante, lourde de parfums et d'opulence de l'Asie. Pourtant, le survol devient peu à peu navigation, qui n'est pas entièrement dénuée de points de repères « réalistes » :

Doch kennt die Inseln der Schiffer.

Und da ich hörte

Der nahegelegenen eine

Sei Patmos,

Verlangte mich sehr,

\footnotetext{
${ }^{6}$ Z. I. Siaflékis, La Relation comparative, Interférences et transitions dans la modernité littéraire, « Transparences méditerranéennes : symbolique insulaire et espace poétique », Paris, L’Harmattan, 2004, p. 30.

${ }^{7}$ Un trajet à considérer dans son unité et son apogée et non pas en étapes discontinues. Voir à ce sujet Martin Heidegger, Approche de Hölderlin (1951), traduit par H. Corbin, M. Deguy, F. Fédier et J. Launay, Gallimard, collection TEL, 1973, 2001, p. 115 : « on peut bien parler d'un chemin de Hölderlin mais non pas d'un tournant. Hölderlin n'a fait que trouver, chemin faisant et seulement ainsi, ce qui lui était propre et ce vers quoi il n'a jamais cessé de se tourner. Sur ce chemin, c'est tout le savoir de la vérité propre à l'hellénisme, au christianisme et à l'Orient d'une façon générale, qui est lui-même en marche. Les divisions en zones et époques dont use habituellement la réflexion historique, deviennent ici caduques ».
} 
Dort einzukehren und dort

Der dunkeln Grotte zu nahn. [...] Gastfreundlich aber ist

Im ärmeren Hauße

Sie dennoch

Und wenn vom Schiffbruch oder klagend

Um die Heimat oder

Den abgeschiedenen Freund

Ihr nahet einer

Der Fremden, hört sie es gern, und ihre Kinder

Die Stimmen des heißen Hains,

Und wo der Sand fällt, und sich spaltet

Des Feldes Fläche, die Laute

Sie hören ihn und liebend tönt

Es wieder von den Klagen des Manns.

[Mais le marin connaît les îles.

Et quand j'ai su

Que l'une des voisines

Etait Patmos,

Grand fut mon désir

$\mathrm{D}^{\prime} \mathrm{y}$ débarquer et là

M'approcher de la sombre grotte. [...] Accueillante elle est

En plus pauvre maison

Quand même

Et si naufragé ou se lamentant

Pour son pays ou un

Ami perdu

L'aborde un

Etranger elle prête l'oreille, et ses enfants,

Les voix du brûlant bocage,

Et d'où le sable s'éboule et se craquelle

L'étendue des champs les bruits

L'entendent et font écho

Tendrement à la plainte de l'homme. $]^{8}$

Cette posture de l'arrivant qui décode le sens d'un paysage est souvent accentuée dans les analyses de Hölderlin par M. Heidegger, d'autant plus que le poète s'inspire parfois des sentiments et des images qui naissent du retour au pays natal. Ainsi, « hommes et choses du pays natal ont un air de famille. Et pourtant ce n'est pas encore cela; ils se referment sur ce qu'ils ont de plus propre. [...] L'arrivant demeure donc encore quelqu'un qui doit chercher. Néanmoins, ce qu'il cherche

\footnotetext{
${ }^{8}$ F. Hölderlin, p. 826-7.
} 
vient déjà à sa rencontre ; cela est proche $» .{ }^{9}$ Dans le cas de Patmos, le passage obligatoire par les «portes de l'Asie » conduit, inévitablement, à un premier temps d'étrangeté, de dépaysement. C'est donc par une série d'associations d'idées et d'épisodes que l'île, à la fin de l'hymne, ne sera plus vue comme un territoire à explorer mais comme le théâtre universel qui permettra à ses acteurs, du moins au poète / pèlerin, de faire retentir le « chant allemand », promesse du dernier vers.

Patmos est donc le troisième espace que le poète décrit : après l'Europe et l'Asie, voilà un paysage fragmentaire, certes, et impressionniste, dont la représentation semble puiser aux sentiments de ce voyageur potentiel, mais qui est associé à jamais à un acte d'hospitalité unique : un jour, l'île prit soin « de l'aimé de Dieu, / Du prophète, qui avait en l'heureuse jeunesse / Accompagné / Le fils du TrèsHaut ». Et à partir de là, dans un flux ininterrompu d'épisodes poétisés, facilité par l'écriture succincte, elliptique, du dernier Hölderlin, nous suivons la Cène, la Pentecôte et l'Ascension, l'abandon des hommes sans image tangible de Dieu, l'espoir et la certitude de la réapparition du divin triomphant, comme si toute cette longue histoire avait comme cadre spatial Patmos, qui ne serait plus liée uniquement à l'événement de l'Apocalypse, mais à l'écartèlement tragique d'une âme collective qui, dans un premier temps, était digne de regarder de près le Fils de Dieu mais qui, peu à peu, est contrainte de concevoir mentalement et d'interpréter les traces invisibles du sacré. ${ }^{10}$

La poésie de Papaditsas et de Gaspar est évidemment dénuée d'une telle narrativité. Pourtant, n'est pas totalement absente une mention furtive de la direction de rapprochement ou d'éloignement de l'île, ce qui fait de cette entité géographique l'aimant fantasmatique qui structure les aventures de l'esprit, de cette «dianoia » de Papaditsas ou de l'existence apparemment aplanie de l'être dans le quotidien, chez Lorand Gaspar. Ainsi, le premier poème du recueil A Patmos est visiblement narratif :

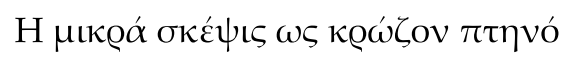

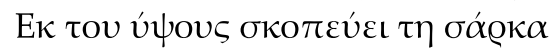

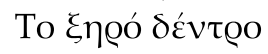

\footnotetext{
${ }^{9}$ M. Heidegger, Approche de Hölderlin, p. 16.

${ }^{10}$ En d'autres termes Jean, l'aimé de Dieu, entretient une relation à la fois métonymique et métaphorique avec l'endroit géographique et le contenu du poème : métonymique, puisqu'il fut le premier hôte de l'île, celui qui a scellé le caractère hospitalier et sacré de Patmos que le poète est sur le point de déchiffrer. Métaphorique, puisqu'il symbolise une histoire passée dont il fut le témoin oculaire, celle de la vie de Jésus. Toutefois les deux aspects sont tellement serrés que le lecteur peut à peine les dissocier, à peine situer Jérusalem dans son antériorité logique par rapport à Patmos dans la vie de Jean. D'où cette impression que Patmos devient l'encadrement non seulement de la mort et de la résurrection d'un dieu, mais aussi le cadre spatial de toute l'aventure de l'humanité.
} 


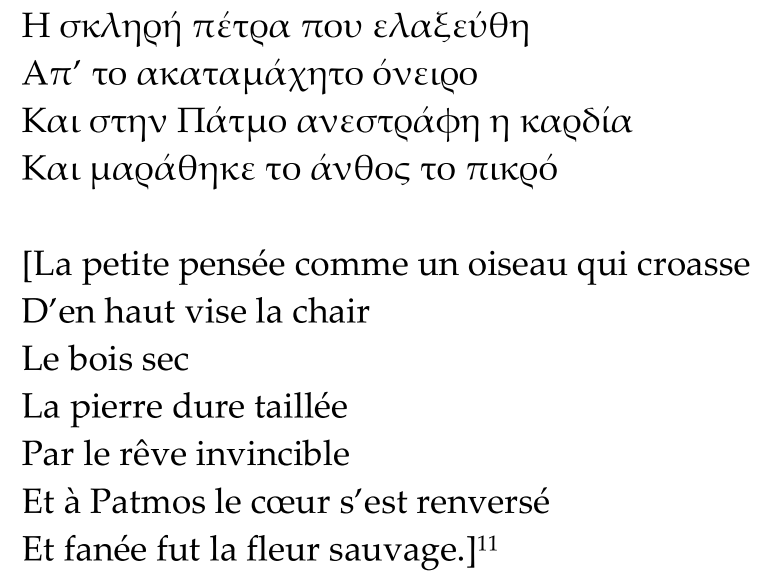

Pourtant, cette pensée survolant la surface de l'île s'en rapproche progressivement et, à travers un système compliqué d'axes reliant le haut et le bas se produit le véritable événement apocalyptique :

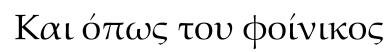

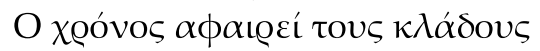

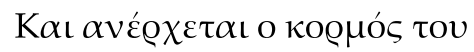

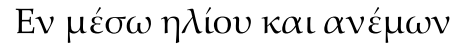

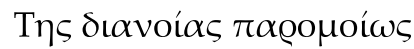

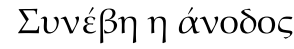

[Et de même que le temps

Enlève les branches du palmier

Et que son tronc monte

Dans le soleil et les vents

De même se produisit

L'élévation de l'esprit.] ${ }^{12}$

Suivent les épisodes, au sens le plus abstrait du terme, de cette élévation et des enjeux qu'elle comporte :

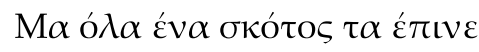

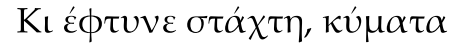

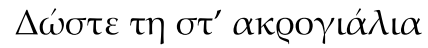

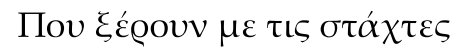

N $\alpha \zeta \omega \gamma \varrho \alpha \phi i ́ \zeta o v v$ to $\alpha \chi \alpha v \varepsilon ́ \varsigma$

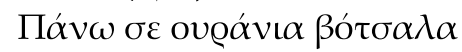

${ }^{11}$ D. P. Papaditsas, A Patmos, p. 23.
${ }^{12}$ D. P. Papaditsas, A Patmos, p. 24. 
[Les ténèbres aspiraient tout

Et crachaient des cendres. Vous, vagues de la mer,

Donnez-les aux côtes

Qui savent dessiner, avec des cendres, l'infini

Sur des cailloux célestes. $]^{13}$

L'élévation de la pensée pallie donc un premier temps, temps de la vanité engloutissante, temps de «Cimetières / Avec la sensation parfumée du disparaître, / Avec comme seul horizon/ La ligne de la douleur et les rêves suspendus ». ${ }^{14}$ Quand survient ce bouleversement dévastateur et régénérateur à la fois, le paysage insulaire y participe en offrant à l'expression du poète / témoin des éléments codifiés de sa constitution géographique, double, elle aussi, unique et universelle.

Patmos de L. Gaspar transforme les bribes de récit contenues dans les Carnets en unités compactes, parsemées dans les poèmes comme s'il s'agissait d'attaches, aussitôt diluées, avec l'univers insulaire. Cela produit, à différents endroits du texte, une progression du détail instantané vers un espace agrandi et intériorisé et de là vers une synesthésie elliptique :

La pêche fut bonne cette année je me souviens de la peur dans les fonds, le combat obscur, la lueur clouée, un timbre éteint dans la musique ${ }^{15}$

Ailleurs, le présent pittoresque s'agrandit en perception du temps mythique :
un olivier noue et dénoue
son obscur désir de clarté
odeur de café sous le platane
un bleu brodé de petites fumées
de mélancoliques fins du monde
Ah! Hélène, folle Hélène ! 16

Ou bien des traits typiquement associés au paysage grec, sur la base d'une conception généralisante, incorporent la présence, le sentiment et la parole du poète dans une coexistence telle que l'attribution de doublets émotifs reste indécise : est-

\footnotetext{
${ }^{13}$ D. P. Papaditsas, A Patmos, p. 29.

${ }^{14}$ D. P. Papaditsas, A Patmos, p. 26.

${ }^{15}$ L. Gaspar, Patmos, p. 9.

${ }^{16}$ L. Gaspar, Patmos, p. 16.
} 
ce la manifestation d'un paysage humanisé ou bien la perception de l'espace selon la projection de l'état d'âme de l'observateur?

Tout le rayonnement de midi moulu dans une poussière d'eau

le vent souffle quand il veut dans nos mots dans nos gestes

brouillant là, éclairant ici

sans distinction de joie de douleur ${ }^{17}$

Pour reprendre ici l'expression utilisée par Elisabeth Démiroglou qui réfléchit sur «la Méditerranée comme réponse à la sollicitation d'un paysage intérieur dans Egée - Judée », dans les deux manifestations modernes de Patmos que nous étudions, la caractéristique de l'expression poétique consiste dans le passage « de la morphologie à l'ontologie »: «Si la Méditerranée, écrit-elle, est d'abord appréhendée en tant que contexte perceptible, elle est ensuite transformée en espace - temps immatériel, en 'une configuration matricielle, une sorte d'invariant commun à la diversité des textes'». ${ }^{18}$ Paysage simplifié jusqu'à ses composantes élémentaires, paysage sur lequel se projette le corps et qui devient lui-même corps à palper ou à disséquer : aussi, malgré la lumière et la transparence qui s'en dégagent, englobe-t-il la peur viscérale atemporelle :
c'est nuit encore dans le ciel
pourtant au ras des eaux les vents
déshabillent les fonds de la pensée 19
encore le matin, la bourrasque levée
je ne sais où sans seuil et sans porte 20

Ainsi, le mythe de Patmos n'est pas nécessairement - ou n'est pas seulement l'empreinte du grand événement biblique de l'Apocalypse, dans le sens de la prophétie liée à la fin du monde et à la domination définitive du Dieu chrétien sur le Mal. Si toutefois nous isolons l'acte de voir, de comprendre et de propager par la parole et l'écriture, acte que devra accomplir Jean en s'adressant aux sept églises de

\footnotetext{
${ }^{17}$ L. Gaspar, Patmos, p. 24.

${ }^{18}$ Elisabeth Démiroglou, «La Méditerranée comme réponse à la sollicitation d'un paysage intérieur dans EgéeJudée et d'autres écrits de Lorand Gaspar ", dans Méditerranée : Ruptures et Continuités, Actes du Colloque tenu à Nicosie les 20-22 octobre 2001, Université Lumière Lyon II, Université de Chypre (sous la direction de May Chehab, Yannis Ioannou, Françoise Métral), Travaux de la Maison de l'Orient Méditerranéen no 37, Lyon, 2003, p. 124.

${ }^{19}$ L. Gaspar, Patmos, p. 25.

${ }^{20}$ L. Gaspar, Patmos, p. 26.
} 


\title{
Lito loakimidou
}

l'Asie au début de l'Apocalypse ou bien en avalant le petit livre que lui tend l'ange puissant qui descendait du ciel, nous nous trouvons au cœur de cette entité antinomique que devient «l'expérience de Patmos » à travers la poésie : en réalité, pas une des trois versions retenues ici qui ne parle d'une composition de l'univers et du sacré qu'il contient, mais d'une composition contrastée, antithétique, comme si l'élévation de la pensée à l'état de l'esprit fertile et énergique, cette «dianoia » de Papaditsas, ne pouvait résulter que de cette expiation moderne que constitue la traversée d'un univers fondamentalement binaire, déchiré et divergent. Que ce soit l'antinomie du statut de Dieu (ou du sacré en général) chez Hölderlin :

\author{
Nah ist \\ Und schwer zu fassen der Gott. \\ Wo aber Gefahr ist, wächst \\ Das Rettende auch. \\ [Proche \\ Et dur à saisir, le Dieu. \\ Mais dans le danger crô̂t \\ Ce qui sauve aussi] ${ }^{21}$
}

Ou bien le double mouvement du regard averti chez L. Gaspar, un regard capable non seulement de passer du macrocosme à l'infiniment petit mais surtout de saisir et d'exprimer la fusion réciproque de l'un dans l'autre :
la paille sous les doigts
craque dans la boue
d'un continent de nuit 22

Patmos mythifiée exige cette acrobatie mentale. L'enjeu est capital : de la masse chaotique du monde surgit l'intentionnalité du paysage, l'appel d'un sens à révéler :

Dans la masse compacte d'une colline déjà presque entièrement sombre,

lisons-nous dans les Carnets de Patmos,

\footnotetext{
${ }^{21}$ F. Hölderlin, p. 824-5. Heidegger fait glisser l'antinomie du côté de l'oxymore, qui contient cependant toute la sagesse du message : «Proche est et difficile à saisir, le Dieu. Cet « et » signifie : et pour cette raison. Le Dieu est trop proche pour être facile à saisir. [...] Trop proches, venant trop près, sont les dieux advenant en direction du poète, sur lui - en venue à sa rencontre. Manifestement cette advenue dure un long temps; elle est pour cette raison plus pesante et donc plus difficile à dire que la présence achevée ». (M. Heidegger, Approche de Hölderlin, p. 247).

${ }^{22}$ L. Gaspar, Patmos, p. 22.
} 
deux maisons, à des hauteurs différentes, les deux notes d'une quinte, qui s'enfoncent de plus en plus dans la masse obscure des rochers, y creusant deux puits carrés d'où sourd assez de blancheur pour que mon œil voie le noir de la nuit

et ailleurs :

Dans le tas de pierres brisées, ramassées au hasard, le maçon cueille celle qui s'ajuste, celle qui remplit exactement l'espace vide préparé par les précédentes. Cette pierre apparemment informe, mal définie, raboteuse, devient l'évidence. ${ }^{23}$

Certes, dans les Carnets, le lecteur peut suivre de plus près la métamorphose du monde insulaire en faisceau de sens universel. Mais dans le recueil de Patmos, le faisceau est déjà là et s'offre à la conquête de la voix intime qui le lit : et, comme il arrive très souvent dans le cas d'une poésie à la fois sobre et dense en images, elliptique et éloquente à la fois, il faut décomposer avant de parvenir à l'idée d'ensemble, passer par exemple de l'idée d'évidence à l'image de l'habitant de Patmos qui va à la pêche ou bâtit sa maison blanche, pour être enfin capable de recomposer l'idée d'ensemble, les péripéties d'un sacré opaque parce que quotidien.

Dans le cas de Papaditsas, déjà dans le Prologue s'éclaire la stylistique particulière de l'antinomie, qui va jusqu'à l'oxymore, qui rend désuète la perception raisonnée : il est question d'expérience intériorisée de l'espace infini et du temps éternel, deux "notions si proches et si éloignées de nous qu'elles nous font peur, nous anéantissent et nous constituent en même temps ». De plus, le vécu de Patmos est sublimé en un état «d'inexistence et en même temps d'existence douloureuse, de silence et de parole absolue, ni moyen ni mode d'expression, mais composante consubstantielle à l'être et au devenir poétiques». Ainsi, l'univers poétique de Papaditsas est plein de couples opposés, qui se déploient sur la tonalité apocalyptique de la décomposition, du tremblement de terre et de la circularité du mouvement universel :

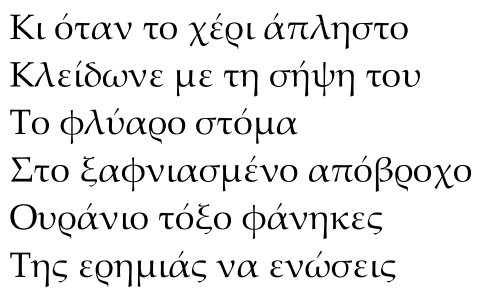

${ }^{23}$ L. Gaspar, Feuilles d'observation, p. 74-75. 


\section{Lito loakimidou}

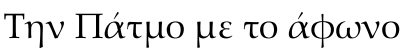

[Et quand la main, avide Verrouillait par sa pourriture

La bouche bavarde

Dans l'étonnement d'après la pluie

Arc-en-ciel tu apparus

Pour lier Patmos du désert

A ce qui est sans voix ] ${ }^{24}$

Et ailleurs :

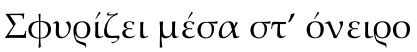

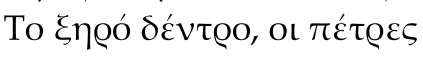

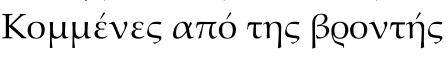

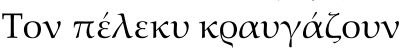

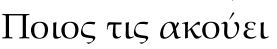

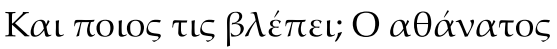

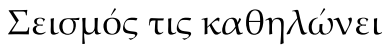

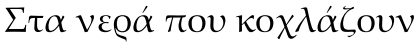

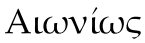

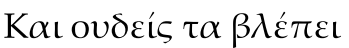

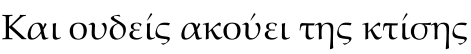

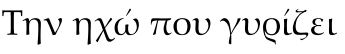

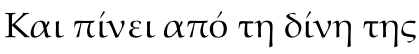

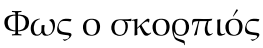

At $\omega v i ́ \omega s$

[Dans le rêve siffle

L'arbre sec, les pierres

Taillées par la hache du tonnerre

Hurlent

Qui les entend?

Et qui les voit? Le séisme immortel

Les immobilise dans les eaux bouillantes

Eternellement

Et personne ne le voit

Et personne n'entend l'écho de l'univers

Qui tourbillonne.

${ }^{24}$ D. P. Papaditsas, A Patmos, p. 30. 
Dans ses remous,

Le scorpion boit de la lumière

Eternellement.] ${ }^{25}$

Il s'agit enfin d'une expérience qui ne se limite pas à la manière inusitée d'englober l'objet extérieur mais qui produit la transsubstantiation de l'être humain. La vue troublée, l'optique aliénée sont exprimées par la célèbre formule de la Première Epître aux Corinthiens : ôs di'esoptrou : «A présent, nous voyons dans un miroir et de façon confuse » :

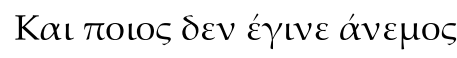

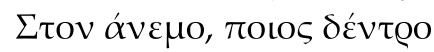

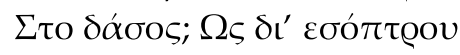

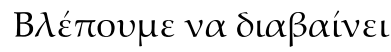

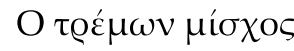 \\ [Et qui ne devint vent \\ Dans le vent, qui arbre \\ Dans la forêt? \\ Comme à travers un miroir, \\ Nous voyons passer \\ La tige frémissante.] ${ }^{26}$
}

En 1984, D. Papaditsas et Héléni Ladia, traducteurs en grec moderne des Hymnes orphiques et des Hymnes homériques, accentuent, dans leur introduction au premier des deux ensembles, leur confrontation pénible à l'adjectif « allotriomorphodiaitos », adjectif trimorphe, telle une autre Hécate aux trois visages, qui, appliqué à la nature orphique, signifierait: celui qui réapparaît toujours sous une forme différente. Depuis longtemps, le poète est marqué par la signification mais aussi par la forme condensée de ce mot, à tel point qu'il lui accorde la place de l'exergue de son poème "Durée», ouverture du recueil homonyme (1972) qui contient aussi une nouvelle version tardive de A Patmos. Même si l'enrichissement adjectival du texte de 1964 est plus modéré, il est clair que Papaditsas porte déjà le sceau de ce qu'il appellera «l'archipel» des mots et des fragments anciens, en privilégiant, toujours par le biais d'une expression antithétique, l'image d'un univers qui tourbillonne sempiternellement : en effet, des vers orphiques «A Ouranos » ou «A Zeus porteur du tonnerre» peuvent être lus en filigrane au-dessous de la surface du texte moderne.

${ }^{25}$ D. P. Papaditsas, A Patmos, p. 32.

${ }^{26}$ D. P. Papaditsas, A Patmos, p. 33. 
Dans son Prologue à Poésie 1 (regroupant les recueils de 1943 à 1961), un texte écrit lui aussi à Patmos en 1962, Papaditsas esquisse les conditions de réception du message poétique contemporain: il parle d'une procédure de transmission s'effectuant en sens inverse par rapport au processus de la création: "Dans les deux cas se met en marche une sorte d'automatisme, même lorsque tout se construit sur un plan préalable, à condition que l'élément à transmettre se forme et se voie de manière poétique. Aujourd'hui, il n'y a plus de lecteurs de poésie, il y a les poètes qui la composent et les poètes qui la reçoivent ».27 Patmos mythifiée est donc l'espace qui accueille et sous-tend l'expérience de l'homme confronté à l'expérience capitale de l'oscillation d'un sens fugitif, équivoque et tremblotant. Il ne s'agit pas d'une énigme dont la solution ouvrirait la porte du monde des élus, des sages. Il s'agit d'une élaboration douloureuse de la conscience d'être à la recherche d'une réponse, $d^{\prime}$ 'un axe structurant le monde, il s'agit, en d'autres termes, de l'expérience mythique par excellence. Patmos relève donc de ce mythique dispersé dans le monde, d'autant plus que l'homme qui pose et qui se pose la question va, à l'image de Jean, commettre l'acte fondateur d'écrire. Ou plutôt la tâche fondatrice d'écrire, comme chez Hölderlin, où le doublet interpréter / chanter par la poésie devient réponse collective («le chant allemand», dit-il) à toute l'expansion culturelle du phénomène religieux. Comme le note Geneviève Bianquis, dans Patmos « se trouvent réconciliées les deux religions qui se sont disputé le cœur de Hölderlin. Elles fusionnent dans l'image d'un Christ hellénistique, plus héros et demi-dieu que rédempteur souffrant et mourant, un peu asiatique aussi à la façon de Bacchus, et complètement intégré à la grande histoire divine de l'humanité ». ${ }^{28}$ Cependant, le mythème le plus important dans ce récit consiste dans la réaction du visiteur de Patmos, un visiteur unique et multiple à la fois, capable de se remémorer une première étape de l'apparition du sacré, une époque de proximité, de visibilité sans obstacle de l'entité divine, soulignée par un champ sémantique très insistant chez Hölderlin : " accompagné le fils du Très-Haut, en inséparable », "eux s'étaient assis ensemble», "ne voulaient pas se séparer du visage du Seigneur », "c'était une chose ancrée comme feu dans le fer », "à leur flanc marchait l'ombre de l'Aimé » : époque bénite, à laquelle succède la dispersion du sacré, une sorte de Babel renouvelée et un univers désorienté :

Wenn aber stirbt alsdenn

An dem am meisten

Die Schönheit hieng, daß an der Gestalt

Ein Wunder war und die Himmlischen gedeutet

\footnotetext{
${ }^{27}$ D. P. Papaditsas, Poésie 1, Athènes, éditions Stigmi, 1985, p. 7.

${ }^{28}$ Hölderlin, Poèmes / Gedichte, introduction, traduction, notes par Geneviève Bianquis, Aubier, éditions Montaigne, 1943, p. 47-48.
} 
Auf ihn, und wenn, ein Rätsel ewig füreinander

Sie sich nicht fassen können

Einander, die zusammenlebten

Im Gedächtniß [...] und selber sein Angesicht

Der Höchste wendet

Darob, daß nirgend ein

Unsterbliches mehr am Himmel zu sehn ist oder

Auf grüner Erde, was ist dies?

[Mais quand vient à mourir

Celui, par-dessus tous

Qui portait la beauté, au point que sa figure

Etait merveille et que les Célestes l'ont marqué

D'un signe, quand énigme à jamais l'un pour l'autre

Ne peuvent plus s'entretoucher

Ceux qui vivaient ensemble

Dans le souvenir [...] et c' est la face même

Du Très-Haut qui se

Détourne, au point que nulle part

Plus rien d'Immortel n'est visible au Ciel ou

Sur la verte Terre, qu'est-ce là ?] ${ }^{29}$

Et ce n'est que dans une troisième étape, celle où le visiteur de l'Asie s'arrête à Patmos, qu'il est question de représenter, après l'avoir conçu et interprété, le sacré désormais intangible: les Ecritures, mentionnées dans les dernières strophes de l'Hymne, ne suffisent pas. L'expression poétique devra incorporer le message syncrétique de l'espace même de Patmos: croisée de continents, suite ininterrompue, fluidité du transcendantal :

$\mathrm{Zu}$ lang, zu lang schon ist

Die Ehre der Himmlischen unsichtbar. [...]

Wir haben gedienet der Mutter Erd'

Und haben jüngst dem Sonnenlichte gedient,

Unwissend, der Vater aber liebt,

Der über allen waltet,

Am meisten, daß gepfleget werde

Der veste Buchstab, und Bestehendes gut

Gedeutet. Dem folgt deutscher Gesang.

[Trop longtemps, trop longtemps déjà

La gloire des Célestes nous est opaque [...].

\footnotetext{
${ }^{29}$ F. Hölderlin, p. 830-831.
} 


\section{Lito loakimidou}

Nous avons servi la Terre-Mère

Et naguère servi le Jour du Soleil,

Ignorants, mais le Père aime,

Lui qui règne sur tout,

Avant tout que l'on cultive

La lettre ferme et que soit bien ce qui demeure

Interprété. Voie d'un chant allemand.] ${ }^{30}$

Une réflexivité pareille n'est jamais explicite dans A Patmos de Papaditsas. Elle transparaît pourtant dans les Deux interprétations, de 1966, où l'expression adoptée insinue le parallélisme entre le visiteur moderne de la Grotte et la posture de l'Evangéliste : on y trouve des poèmes tissés autour de «ce que j'entendis », "ce que je vis », « ce qui vint à moi », « ce qui revient comme un brouillard », " ce qui est comme un écho incandescent», «ce qui chanta tout comme un oiseau», l'ensemble poétique se terminant par la déclaration suivante :

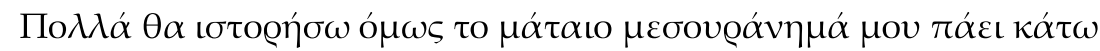

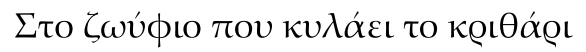

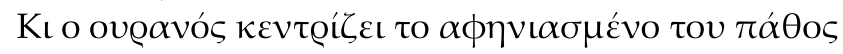

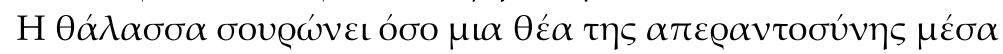

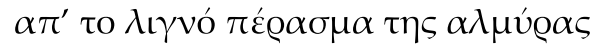

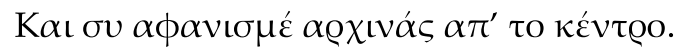

[Ma narration sera longue, pourtant mon vain zénith se penche

$\mathrm{Au}$-dessus de la bestiole qui roule le grain

Et le ciel talonne sa passion enragée

La mer se fronce telle une vue de l'infini à travers l'étroit passage du sel

Et toi, dévastation, tu commences par le centre.] $]^{31}$

C'est pourtant dans le poème en guise de conclusion du recueil $A$ Patmos que nous trouvons l'implication fertile de l'acte de Jean, de la rédaction poétique et du lecteur :

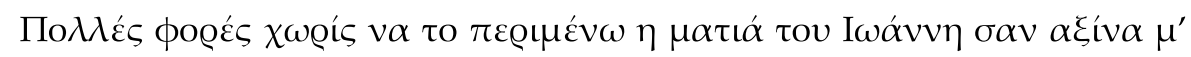

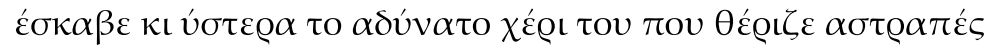

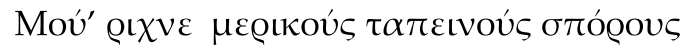

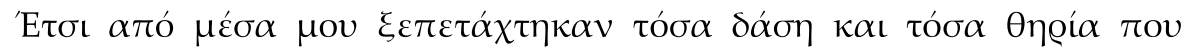

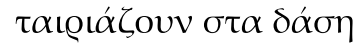

\footnotetext{
${ }^{30}$ F. Hölderlin, p. 834-5. La traduction française des deux derniers vers peut prêter à confusion : il faut prendre l'ensemble de l'expression «ce qui demeure » comme sujet de «soit bien interprété », en d'autres termes, ce qui demeure, le demeurant, la quintessence immobile doit devenir l'objet d'un transfert poétique dans le domaine de l'intellect et de l'art en même temps. Bien sûr le poète a un rôle nodal dans ce passage d'image en image, de siècle en siècle, dans le respect fertile de « la lettre ferme » et de « ce qui demeure ».

${ }^{31}$ D. P. Papaditsas, Poésie 2, p. 58.
} 


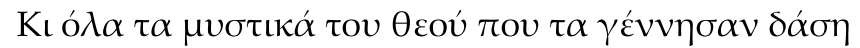

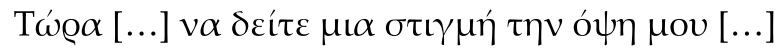

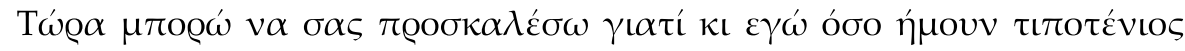

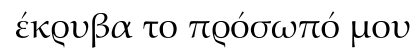

[Souvent, sans que je m'y attende, le regard de Jean creusait en moi comme une pioche et sa main frêle qui moissonnait des éclairs

Semait en moi quelques grains humbles

Ainsi, naquirent de moi-même tant de forêts et tant d'animaux sauvages qui

s'y rattachent

Et tous les secrets divins nés dans la forêt.

Maintenant, $[\ldots]$ en un clin d'œil vous pouvez voir ma face $[\ldots]$

Maintenant je peux vous inviter: tant que je n'étais rien, je cachais mon visage.] $]^{32}$

C'est dans le recueil de Lorand Gaspar que le vécu, pittoresque et délimité ou sublimé et généralisé, associe Patmos à ce qu'on pourrait désormais appeler «mythe de l'écriture » : l'écriture, non pas seulement comme exercice de l'esprit et du cœur ou comme un mouvement fervent qui renouvelle ce qui se passa dans la Grotte, une main qui met en lumière ce qui doit être vu, mais aussi la substance matérielle, le papier, l'encre sacralisés. Ce n'est donc pas la dimension temporelle qui synthétise les couches différentes du passé, mais aussi la dimension spatiale qui s'étend jusqu'à la Chine, dont le nom, proféré dans ce contexte, condense les outils de base :
Comme elle nage, la lumière!
légère et souple entre les dents
de dragons s'ébattant dans les gouffres
d'une Chine de l'âme inoubliée [...]
et l'aile noire du pinceau
frôla le vent qui bouge à peine
les bambous sur le muet, muet
papier de $\mathrm{Chu} \mathrm{Ta}^{33}$

L'allusion à ce maître de l'encre du XVIIe siècle, qui a vécu une vie d'ermite et qui cessa de parler, condamné à un mutisme peut-être volontaire, ainsi que la mention de dragons et des «flocons brûlants de leur souffle » montrent à quel point des détails signifiants des deux univers peuvent s'enchevêtrer, voire se substituer l'un à l'autre. Dans un univers où «trois vieilles vêtues de noir» deviennent «chœur

\footnotetext{
${ }^{32}$ D. P. Papaditsas, Poésie 2, p. 35-36.

${ }^{33}$ L. Gaspar, Patmos, p. 10.
} 


\section{Lito loakimidou}

antique qui salue sur le seuil », puis «Erinyes » qui chantent, dans un décor intime où les « ombres blanches qui passe[nt] dans la chaux » font penser à Ulysse et « aux sanglots des hommes quand l'aveugle annonce le retour difficile ${ }^{34}$, nous voyons circuler, ne serait-ce que furtivement, des figures lointaines, dont le destin, schématisé en quelques vers, se dessine dans les mêmes éléments et s'exprime par les mêmes mots que ceux qui correspondent au paysage grec :

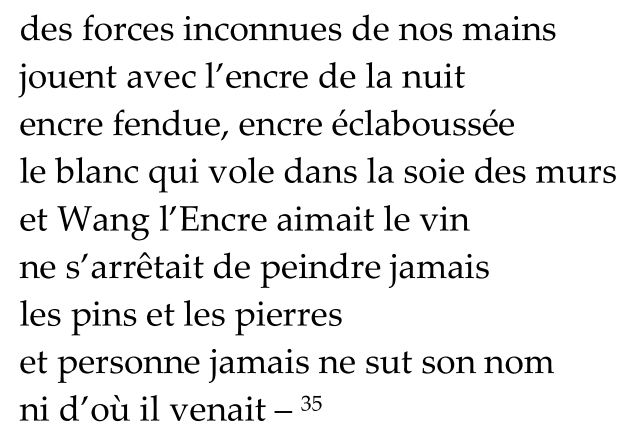

Pourquoi cette insistance sur la proximité de deux mondes qui, de prime abord, semblent dépareillés ? Peut-être parce que cette encre est la matérialisation de la parole qui essaie de concevoir, de recréer l'ordre de l'univers :

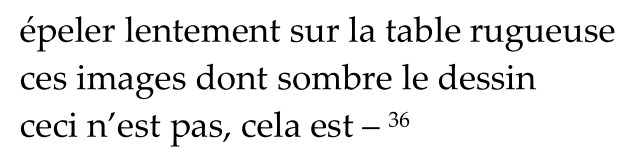

Peut-être aussi parce que sur ces œuvres d'art, c'est encore l'œil qui s'exerce à déchiffrer

la dentelle de l'eau, la vapeur qui roule

sur le bord de nos pages désertées ${ }^{37}$

avec cette belle paronomase des «plages » en "pages», qui substitue un objet venant du monde de l'écriture à l'un des attributs les plus emblématiques du monde insulaire, mais aussi de l'aventure du voyageur en mer.

Peut-être enfin parce que le pinceau, dont Lorand Gaspar a loué par ailleurs la grâce, la fermeté, ces " cils longs des pins du jardin un matin après la pluie », ces traits d'encre qui captent leurs propres mouvements lumineux et laissent sur la

\footnotetext{
${ }^{34}$ L. Gaspar, Patmos, p. 18.

${ }^{35}$ L. Gaspar, Patmos, , p. 15

${ }^{36}$ L. Gaspar, Patmos, p. 12.

${ }^{37}$ L. Gaspar, Patmos, p. 19.
} 
page « une gouttelette de feu transparent, d'où jaillissent des rayons verts et rouges, joyaux des Mille et Une Nuits ", ce pinceau, ainsi que les rides infinitésimales de la couleur liquide, donne un sens ferme à l'effort désespéré de ${ }^{38}$

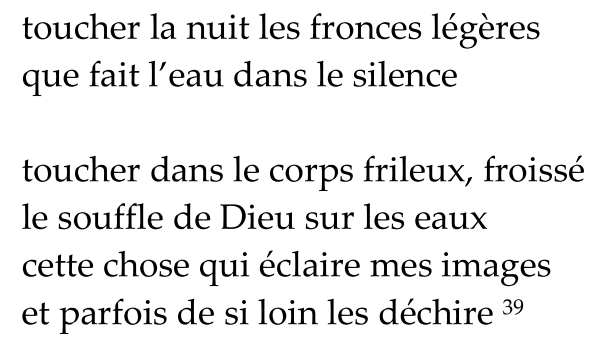

Les trois expériences poétiques étudiées ici métamorphosent donc l'Apocalypse et Patmos en mythe de l'écriture, sur un ton qui va de la célébration glorieuse à l'intimité lyrique; pour ce faire, elles se servent d'une expression et d'une couche de symboles syncrétiques et parfois hétérogènes: de Hölderlin, qui mélange la longue série des manifestations du divin avec l'histoire de Jésus et la terre de Patmos, à Papaditsas, qui se sert de la stylistique particulière des hymnes orphiques et homériques pour parler d'un bouleversement apocalyptique de l'esprit et de la création verbale, jusqu'à L. Gaspar, poète qui fait coexister la grécité antique et l'univers de l'orient, l'aspect familier d'une île aimée, la tradition chrétienne d'un souvenir fondateur et l'universalité de toute manifestation artistique produite par la main qui se met à retracer la vision du moi projeté sur l'espace, la liste est longue des attributs qui viennent s'enchevêtrer et donner une idée de ce jeu de miroirs illimité que devient la signification complexe de l'entité mythique.

Voyager vers Patmos, se rapprocher peu à peu de l'île, prendre en charge le poids mental et psychologique de cette entreprise ; devenir conscient d'un espace-temps qui apparaît d'abord comme déréglé, antinomique et qui laisse à peine deviner la sagesse de son syncrétisme culturel mais aussi le syncrétisme des moyens de le décoder; saisir surtout l'intermittence des apparitions du sacré, les différents niveaux de sa manifestation; recommencer, enfin, le geste rédacteur de Jean, non pas dans une imitation sèche et conventionnelle, non pas en vue d'un climat apocalyptique d'une portée contestable, mais en vivant l'écriture comme une cosmogonie de la parole, du sens et du sacré, tels sont les mythèmes centraux de cette aventure de l'être et de la création poétique. Couronnant la période des grands hymnes chez Hölderlin, constituant un nouveau point de départ, une pierre de touche pour la création plus récente de Papaditsas ou bien une référence constante

\footnotetext{
${ }^{38}$ L. Gaspar, Feuilles d'observation, p. 123-124.

${ }^{39}$ L. Gaspar, Patmos, p. 23.
} 


\section{Lito loakimidou}

dans la conception de l'univers chez L. Gaspar, l'écriture qui prend pour sujet Patmos aboutit à ce mythe tout neuf dont parlait Papaditsas, mais aussi à la longue série des tentatives de mythification d'elle-même. 